Christian Larsen, Claus Hoe Seiding, Christian Teller \& Anders Thorstenson

An inventory control project in a major Danish company using compound renewal demand models

Logistics/SCM

Research Group 


\title{
An inventory control project in a major Danish company using compound renewal demand models
}

\author{
Christian Larsen ${ }^{1}$, Claus Hoe Seiding ${ }^{2}$, Christian Teller ${ }^{2}$, \\ and Anders Thorstenson ${ }^{1 *}$ \\ ${ }^{1}$ Logistics/SCM Research Group, Department of Business Studies \\ Aarhus School of Business, University of Aarhus \\ Fuglesangs Allé 4, DK-8210 Aarhus V, Denmark \\ ${ }^{2}$ The Danfoss Group \\ Refrigeration \& Air Conditioning - Global Logistics \\ Nordborgvej 81, L3-Ø118, 6430 Nordborg, Denmark
}

\begin{abstract}
:
We describe the development of a framework to compute the optimal inventory policy for a large spare-parts' distribution centre operation in the RA division of the Danfoss Group in Denmark. The RA division distributes spare parts worldwide for cooling and A/C systems. The warehouse logistics operation is highly automated. However, the procedures for estimating demands and the policies for the inventory control system that were in use at the beginning of the project did not fully match the sophisticated technological standard of the physical system. During the initial phase of the project development we focused on the fitting of suitable demand distributions for spare parts and on the estimation of demand parameters. Demand distributions were chosen from a class of compound renewal distributions. In the next phase, we designed models and algorithmic procedures for determining suitable inventory control variables based on the fitted demand distributions and a service level requirement stated in terms of an order fill rate. Finally, we validated the results of our models against the procedures that had been in use in the company. It was concluded that the new procedures were considerably more consistent with the actual demand processes and with the stated objectives for the distribution centre. We also initiated the implementation and integration of the new procedures into the company's inventory management system.
\end{abstract}

Keywords: Base-stock policy, compound distribution, fill rate, inventory control, logistics, stochastic processes.

\footnotetext{
* Corresponding author; e-mail: ath@asb.dk, tel: +45-8948 6318, fax: +45-8948 6660
} 


\section{Introduction}

In many standard implementations of inventory control systems continuous demand is assumed, and a service level constraint is applied. Lead-time demand is then usually modelled as a (truncated) normally distributed stochastic variable. The service level constraint is most commonly specified either as a fill rate requirement or as a cycle-stock service-level requirement. This may work well in many settings, particularly for standard high-volume products. However, in some cases this approach is clearly not satisfactory, especially not when demand is lumpy and/or the importance of each customer order is given equal weight. Recently, we have been involved with such a case in our work for one of the largest industrial companies based in Denmark, the Danfoss Group.

The company is involved with research \& development, production, sales and service of mechanical and electronic components for several industries. The Danfoss Group is a leading manufacturer of valves and fluid handling components for HVAC and industrial applications. It has three main business areas: Refrigeration and Air Conditioning (RA), Heating and Water, and Motion Controls. The company's annual turnover in 2005 was approx. 2,200 million EUR, and it employs about 18,200 people worldwide. The RA division, in which this case study was carried out, accounts for approximately $50 \%$ of the total turnover.

At its major distribution centre located in the south of Denmark, the RA division was experiencing typical inventory problems of stock allocation and control, i.e., frequent shortages of certain stock keeping units (SKUs) coupled with a relatively high aggregate level of capital tied up in inventory. The SKUs handled by the distribution centre cover a fairly broad spectrum of different kinds of spare parts, including many with low frequent but lumpy demand. Initially, we identified two important reasons for the stock control problems. First, the demand distribution used for inventory control purposes did not fit well with the empirical demands. Second, the inventory control method that was applied was not consistent with the performance criterion that was used. The first reason is related to the forecasting system, hereby also to data mining, while the second reason is related to the design of the inventory control system. Preferably, these two systems should also be integrated in the total control system. Empirical relevance, consistency of 
the approach and integration of forecasting and inventory control systems then formed the overall objectives for this case study in order to improve the operations of the RA division's major distribution centre. Eventually, the goal is to obtain similar improvements also at the division's other distribution centres worldwide.

In the first phase of the case study, inspired by the inventory control literature on lumpy demand, we focused on compound Poisson distributions to model the empirical demand distributions. In order to obtain a reasonably good fit, the demand data available first had to undergo a simple filtering process. The choice of compounding distribution was also an issue. Starting with the geometric distribution, we identified a menu of discrete compounding distributions to choose from depending on the empirical data estimates at hand for a particular SKU. In a later phase of the case study we also observed that the Poisson process was not a good description of the demand process. A better match could be obtained by assuming more generally a renewal process, and then empirically fitting Erlang- $k$ processes to those SKUs whose demand processes exhibited a more regular pattern.

As regards the performance criteria for the inventory control system, capital tied-up in inventory was obviously an important criterion recognized by the company. However, rather than adopting, as in a standard setting, a fill rate or a cycle service level as the service level requirement, the inventory managers argued that an order fill rate should be applied. In fact inventory service performance was already based on measuring the percentage of complete orders for single SKUs filled on time. To distinguish the order fill rate from the standard, unit-based fill rate we term the latter the volume fill rate to indicate its unit-based nature. The basis for using an order fill rate is the focus on individual customer orders and the distribution centre's ability of filling each of those orders in full from inventory. The focus on customer order fulfilment indicates that equal weight is attached to each order irrespective of its size or volume. This corresponds to a cost of shortages related to the occurrence of a shortage, not its size. Of course, if each customer order were for a single unit, then the order fill rate and the volume fill rate would be identical. However, as noted above, this is not always the case, and customer order may be for several units at a time. Hence, this part of the case study involved development of specifications and algorithms related to the order fill rate to harmonize 
with the RA division's base-stock, inventory control system. Obviously, the specifications and algorithms then also had to be integrated with the empirical demand estimates referred to above.

The remainder of this paper is structured as follows. In Section 2 we give a brief review of some of the relevant literature. Section 3 describes how demand information is used to specify the probability distributions used in the inventory control system. The inventory control system, in particular the specification of base-stock levels in terms of the servicelevel requirement, is outlined in Section 4. This is followed by a discussion of various implementation and application issues in Section 5. Finally, in Section 6 we summarize the results of the project at the present stage, and discuss routes for future developments in order to make the system even more apt to satisfy the needs of the company for a functional inventory control tool.

\section{Literature review}

SKUs with lumpy and low frequent demand are generally more difficult to forecast and control than SKUs having a stable and steady demand (Willemain et al., 2004). Lumpiness refers to the fact that demands are not always unit-sized, and low frequent is sometimes labelled as intermittent. In particular, demand for spare parts is often observed to be characterized by lumpiness and relatively low frequency. Compound Poisson demand processes have been suggested in the inventory literature to model demand having these characteristics.

One of the early contributions on the application of this type of demand process in inventory models is Feeney \& Sherbrooke (1966). Ward (1978) also uses compound Poisson demand in the development of an inventory control model based on a case study. A more recent example is Matheus and Gelders (2000). The newly updated textbook by Axsäter (2006) contains a fairly extensive treatment of demand represented by a compound Poisson process in inventory control. In particular, with reference to Feller (1966), he notes that a non-decreasing stochastic process with stationary and mutually independent increments, which is a common assumption for cumulative demand in inventory control theory, may always be represented as a limit of an appropriate sequence of compound Poisson processes (Axsäter, ibid., p. 77). Renewal compound demand 
processes for inventory control have been treated to a much lesser degree in the literature, as pointed out by Smith and Dekker (1997). They also note that, whereas a base-stock policy is the optimal policy in a standard cost setting under compound Poisson demand (see, e.g., Zipkin, 2000, Ch. 6), this is not true in general for compound renewal demand. Nevertheless, a base-stock policy might be the preferred policy in a practical setting due to its simplicity.

In standard inventory control presentations and applications cumulative lead-time demand is often assumed to be specified by a normal distribution. The gamma distribution is sometimes, as in Burgin (1975) and Tyworth et al. (1996), suggested as an alternative to the (truncated) normal distribution. Alternatively, the negative binomial distribution is suggested to model discrete demand over the lead time. It is well-known that this distribution is obtained in the case where demand is generated by a pure Poisson process, and thus is unit-sized, and where lead-time is gamma distributed (Hadley and Whitin, 1963). For an overview of several other lead-time demand distributions that have been suggested in the literature see Silver et al. (1998).

The choice of compounding distribution for a compound demand process poses an estimation problem. The stuttering Poisson process, i.e., the compound Poisson process with the geometric distribution as the compounding distribution is frequently proposed in the literature. Recently, Johnston et al (2003) found some empirical evidence to support the assumption of using the geometric distribution as the compounding distribution. The geometric distribution is a special case of the negative binomial distribution, which belongs to the family of power series distributions that also includes the binomial, the Poisson, and the logarithmic distributions. Note that if the order arrival process is Poisson and the compounding distribution is logarithmic, then the cumulative lead-time demand follows a negative binomial distribution (Zipkin, ibid.).

Regarding forecasting of intermittent demand for spare parts, see Hua et al. (2007) for a recent overview. Willemain et al. (2004) categorize forecasting methods for lumpy and low frequent demand. Among the categories is Croston's method; See Croston (1972) for the original reference and Johnston and Boylan (1996) for a more recent reference. Underlying Croston's method is the basic idea of decomposition of the demand process 
into demand intervals and demand volumes. This corresponds well with the structure of the compound demand processes referred to above. Park (2007) analyzes different approaches to decomposition of compound cumulative demand distributions. In addition to the order size and order intensity/inter-arrival time components, the lead time component is also considered.

Service levels are used in inventory control systems for performance evaluation and in target setting as substitutes for shortage costs that are hard to estimate. A review of standard service level measures and their relationships to shortage costs and different inventory control policies is provided by Schneider (1981). Another overview is provided

in Tempelmaier (2000). Most textbooks on operations management and/or inventory control include treatments of this topic, because service levels are ubiquitous in practical inventory systems. One of the most commonly used performance measures in inventory control is the (volume) fill rate, defined as the fraction of total demand volume that can be satisfied from inventory without shortages (Silver et al., 1998, p. 245). Song (1998) deals with the composite fill rate in a setting with multiple SKUs. Chen and Krass (2001) considers minimal service level constraints.

Somewhat less common as a performance measure in inventory control is the order fill rate $(O F R)$, specified as the fraction of complete orders that can be filled directly from inventory. Sometimes it is also labelled the line fill rate or the (perfect) order fulfilment rate. In Larsen and Thorstenson (2006) the OFR is specified for a base-stock inventory control system and its relation to the fill rate and to other service measures such as the ready rate (see, e.g., Axsäter, 2006) is analyzed.

\section{Demand information}

Describing the demand process by a probability model is one of the essential inputs in an analytical model of a stochastic inventory control system. Therefore, before developing any kind of mathematical inventory model for practical application, it must first be clarified which sort of demand data that can be extracted from the company's records of demand information. In particular, is the demand data available in a very aggregated form, such that only information about the total demand in, say, a week can be extracted, or is it possible to obtain information about every specific customer order? In this case it 
turned out that we were fortunate to be able to acquire detailed data of the latter form from the company's records. Hence, it was natural to decompose the demand process into two components: the size of an individual customer order (modelled as a positive integer-valued random variable $X$ ) and the time between two consecutive order requests (modelled as a positive continuous random variable $T$ ). The demand information extracted from the company's records then made it possible to fit empirical estimates of the customer order sizes and the times between orders to some alternative theoretical probability distributions for $X$ and $T$.

Before considering the choice of appropriate probability distributions, we took an issue into account that is not strongly emphasized in standard texts on mathematical models for inventory control, like Silver et al (1998) and Zipkin (2000). It is the aspect of advanceorder information. When a request for an order $i$ is received at time point $\tau_{i}$, there is an agreed order delivery lead time $D T_{i}$, so that the customer requires a (physical) delivery at time point $\tau_{i}+D T_{i}$. Usually, in standard texts, it is tacitly assumed that $D T_{i}=0$, implying that customers expect instantly delivery. In Silver et al (1998) it is not mentioned at all that $D T_{i}$ might be positive. In Zipkin (2000) the possibility of a positive $D T_{i}$ is only mentioned in an exercise (Exercise 6.1). However, to some extent the issue has been treated in the literature; See e.g. Hariharan and Zipkin (1995) and Marklund (2006).

Particularly, if the order delivery lead time $D T_{i}$ is longer than the estimated replenishment lead time $L$, then order $i$ is a completely known order, because there is enough time to plan for this order without depending on inventory holding. Hence, there is no need to keep an inventory for known orders to avoid the risk of shortage (only to capture possible economies of scale in ordering; however, this possibility was ruled out in this case by the company's choice of a base-stock ordering system for inventory control). To distinguish the known customer orders from the others, we introduced a simple (and crude) filtering rule: All orders with an agreed order delivery lead time longer than the estimated replenishment lead time of the SKU $\left(D T_{i}>L\right)$ are excluded from the data. Consequently, it is also implied that the known orders should be handled separately from the stochastic inventory control system. 
The remaining data, i.e. data for the (partly) unknown orders are subsequently used for estimation of parameters for the random variables $X$ and $T$. It is of course essential that this filtering process is carried out before parameters of $T$ are estimated. Otherwise, one would exaggerate the intensity of the arrivals of the (unknown) order requests. Concerning the estimation for $X$, one could argue that if there is no significant difference between the sizes of known and unknown orders, then in order to have a larger data set, one should estimate parameters of $X$ on the basis of all order-size information. However, in some cases we observed that the known order-sizes had characteristics that were quite different from the unknown orders. They are often larger and also frequently related to 'box' or package sizes. Because of this possible effect, the parameters for $X$ are also estimated using only the unknown customer order data. As noted above, the filtering rule is crude and any information contained in order deliver lead times $0<D T_{i}<L$ is currently disregarded. We comment on this issue in Section 6.

\subsection{Estimation of the customer order size $(X)$}

To estimate the parameters of the customer order size distribution, we consider an SKU for which there is a record of $n$ orders of sizes $x_{1}, x_{2}, \ldots, x_{n}$. From this we compute the empirical mean, EmpM, and variance, EmpVar:

$\operatorname{EmpM}=\frac{1}{n} \sum_{i=1}^{n} x_{i}$

and

EmpVar $=\frac{1}{n-1} \sum_{i=1}^{n}\left(x_{i}-\text { EmpM }\right)^{2}$.

We then apply one of three alternative theoretical probability distributions to fit data, namely the negative binomial, the binomial and the Poisson distribution. These three discrete distributions belong to the family of power series distributions (PSDs). They are motivated by their computational tractability and by the fact that they allow for different levels of the customer order size variance in relation to its mean. The Poisson distribution is the limiting case of both the negative binomial distribution and the binomial 
distribution, when the variance approaches the mean. The PSD distributions encompass a broad family of unimodal distributions with appealing shapes.

When $X$ follows a negative binomial distribution, the probabilities $P(X=j)$ are given by

$P(X=j)=\frac{(s+j-2) !}{(j-1) !(s-1) !}(1-\rho)^{s} \rho^{j-1} \quad j=1,2, \ldots$,

where the form parameter $s$ is positive (but it does not have to be integer valued), and the probability parameter $\rho$ satisfies $0<\rho<1$. The geometric distribution is the special case, when $s=1$. The mean, $E[X]$, and variance, $V[X]$, of the negative binomial distribution are given by

$E[X]=\frac{s \rho+1-\rho}{1-\rho}$

and

$V[X]=\frac{s \rho}{(1-\rho)^{2}}$

Note that

$V[X]>E[X]-1$.

Therefore, it only makes sense to model customer orders as negative binomially distributed, if

EmpVar $>$ EmpM -1 .

When this relation holds for the SKU, the parameters $s$ and $\rho$ are estimated from the two equations:

$$
\begin{aligned}
& \frac{s \rho+1-\rho}{1-\rho}=\text { EmpM } \\
& \frac{s \rho}{(1-\rho)^{2}}=\text { EmpVar }
\end{aligned}
$$

which have the solution $\hat{s}$ and $\hat{\rho}$ given by 


$$
\begin{aligned}
& \hat{\rho}=\frac{E m p V a r-E m p M+1}{E m p V a r}, \\
& \hat{s}=\frac{(1-\hat{\rho})(E m p M-1)}{\hat{\rho}} .
\end{aligned}
$$

Note also that for the negative binomial distribution

$$
P(X=1)=(1-\hat{\rho})^{\hat{s}}=e^{\hat{s} \ln (1-\hat{\rho})}
$$

and

$$
P(X=j+1)=\frac{\hat{s}-1+j}{j} \hat{\rho} P(X=j) \quad j=1,2, \ldots,
$$

so that the probabilities can be computed recursively.

When $X$ follows a binomial distribution, the probabilities $P(X=j)$ are given by

$$
P(X=j)=\frac{(n-1) !}{(j-1) !(n-j) !} p^{j-1}(1-p)^{n-j} \quad j=1,2, \ldots, n
$$

where the parameter $n$ is positive and integer valued, while the parameter $p$ satisfies $0<p<1$. Its mean and variance are given by

$E[X]=(n-1) p+1$

and

$V[X]=(n-1) p(1-p)$.

Note that

$E[X]-1>V[X]$

Therefore, it only makes sense to model customer orders as binomially distributed, if EmpM-1> EmpVar.

When this relation holds for the SKU, we estimate the parameters $n$ and $p$ by the two equations

$$
\begin{aligned}
& (n-1) p+1=E m p M, \\
& (n-1) p(1-p)=E m p V a r,
\end{aligned}
$$


which have the solution $\hat{n}$ and $\hat{p}$ given by

$\hat{p}=\frac{E m p M-1-E m p V a r}{E m p M-1}$,

$\hat{n}=\frac{E m p M-1}{\hat{p}}+1$

It is required that $\hat{n}$ is an integer. A simple approach is then to use the solution above rounded up to its nearest integer. Thus

$\hat{n}=\operatorname{Int}\left(\frac{E m p M-1}{\hat{p}}+1.99\right)$,

where $\operatorname{Int}(\cdot)$ returns the integer part of the argument. (An alternative and somewhat more sophisticated, but computationally less convenient, approach would be to construct the empirical parameter values from a mixture of two binomial distributions with adjacent values of $n$; See Bradley and Robinson, 2005, where this approach is applied in a basestock inventory control model.)

Note also that for the binomial distribution

$P(X=1)=(1-\hat{p})^{\hat{n}-1}=e^{(\hat{n}-1) \ln (1-\hat{p})}$

and

$P(X=j+1)=\frac{\hat{n}-j}{j} \frac{\hat{p}}{1-\hat{p}} P(X=j) \quad j=1,2, \ldots$,

so that the probabilities can again be computed recursively. The recursion formula automatically facilitates that $P(X=j+1)=0$, when $j=\hat{n}$.

When $X$ follows a Poisson distribution, the probabilities $P(X=j)$ are given by

$P(X=j)=e^{-\delta} \frac{\delta^{j-1}}{(j-1) !} \quad j=1,2, \ldots$

where the parameter $\delta$ is positive. Its mean and variance are given by

$E[X]=\delta+1$,

$V[X]=\delta$. 
Note that

$E[X]-1=V[X]$.

Therefore, it only makes sense to model customer order sizes as Poisson distributed if EmpM - 1 'is close to' EmpVar. Below, it will be explained how the phrase 'is close to' is made operational. When EmpM - 1 'is close to' EmpVar we estimate the parameter $\delta$ by $\hat{\delta}=E m p M-1$

Note further that

$$
\begin{aligned}
& P(X=1)=e^{-\hat{\delta}}, \\
& P(X=j+1)=\frac{\hat{\delta}}{j} P(X=j) \quad j=1,2, \ldots,
\end{aligned}
$$

so that the probabilities can be computed recursively in this case, as well.

When EmpM - 1 'is close to' EmpVar, we have in reality two (or three) distributions to choose between. Therefore, this case can also serve as a test of robustness: Whichever distribution we choose, the control parameters computed and the corresponding service levels should be almost equal.

To make the choice of probability distribution for the customer order size operational, we introduce a tolerance parameter $\gamma$ (typically $\gamma=0.1$ ). The choice of distribution is then decided by the following rule:

If EmpVar $<(1-\gamma)(E m p M-1)$ then choose a binomial distribution

If $(1-\gamma)(E m p M-1) \leq E m p V a r \leq(1+\gamma)(E m p M-1)$ then choose a Poisson distribution If EmpVar $>(1+\gamma)(E m p M-1)$ then choose a negative binomial distribution.

By analyzing the same data set with different values of $\gamma$, we can make the above mentioned robustness test. Setting $\gamma=0$ essentially eliminates the Poisson distribution as an alternative.

In a later version of the model we made an essential modification to the procedure described above. When fitting to a negative binomial distribution, the fitted parameter 
value $\hat{\rho}$ sometimes turned out to be very high, around 0.99 . This implies that our model assumed an order-size distribution with a rather large tail probability, i.e. with a rather high probability that a quite large order will arrive. Consequently, the model proposed a quite large base-stock level. In practice, it was not considered very likely that such a large order should arrive (unexpectedly). Hence, it was felt that the model behaved too conservatively. As a remedy for this an additional input is registered, namely the largest customer order recorded, CosMax (abbreviation for 'customer order size maximum').

$\operatorname{CosMax}=\operatorname{Max}\left\{x_{i}: i=1, \ldots, n\right\}$

The information about CosMax is only considered, if the negative binomial distribution is chosen. In that case we first compute the parameter estimates $\hat{s}$ and $\hat{\rho}$ as explained above. Subsequently, these two values are modified to fulfil the following two requirements:

$\frac{s \rho+1-\rho}{1-\rho}=\operatorname{EmpM}$

and

$P(X \geq \operatorname{Cos} M a x) \leq \varepsilon_{1}$

where $\varepsilon_{1}$ is a small number, typically $\varepsilon_{1}=0.01$. By introducing this extra procedure we often manage to correct for the previously observed conservative behaviour of the inventory control model.

\subsection{Estimation of the time between consecutive order requests (T)}

We now turn to estimation of the second component of the demand process, the time between customer orders. Assume that the $n$ orders were recorded in a time interval of length $\tau$. The customer order intensity (the number of customer order requests per time unit) is then simply estimated as $\lambda=n / \tau$. In an earlier version of the model, it was assumed that the arrival of order requests could be described by a Poisson process. This is a common assumption in many mathematical models of inventory control (see e.g. Axsäter, 2006 for an overview). However, when examining the actual datasets, this was often observed to be a too restrictive assumption. In reality, the arrival pattern was less 
erratic than assumed by our model which cautiously proposed too high base-stock levels. This effect has also been noted elsewhere in connection with inventory control for spare parts (Smith and Dekker, 1997). Therefore, we decided to collect data for an additional input parameter, TBOmin (abbreviation for 'minimum time between orders'), which is the minimum time recorded between two successive order requests. This extra input parameter is used to fit the distribution of $T$ to a $k$-phased Erlang distribution with mean value $1 / \lambda$. The phase parameter is then found as the smallest positive value of $k$ satisfying $P(T \leq T B O \min ) \leq \varepsilon_{2}$,

where $\varepsilon_{2}$ is a small number, typically $\varepsilon_{2}=0.01$. Note that if TBOmin $=0$, then the procedure will generate $k=1$, which implies an exponentially distributed time between orders, i.e. a Poisson process. Thus, the later version of our model is a genuine generalization of the earlier version. The motivation for choosing the Erlang distribution is that it is computationally tractable while being versatile enough to provide a wide variety of shapes.

\subsection{Alternative estimation procedures}

For some of the simple methods used for estimating the demand process there may exist better alternative procedures. Instead of solving an equation system based on point estimates, Maximum Likelihood Estimation (MLE) might have been applied. However, for the case of the negative binomial distribution it would be more involved to do MLE, requiring the use of an optimization method, see Law and Kelton (1991; p. 348). We are not convinced that the final results obtained by using MLE, and the results from using our simpler method would differ significantly in practice. Moreover, in many cases the number of observations is rather small, so the result of any (more or less sophisticated) method should anyway be considered with caution.

The introduction and usage of the additional input parameters CosMax and TBOmin might appear somewhat ad hoc, although they serve the legitimate purpose of identifying outliers and avoiding extreme solutions. Admittedly, this was a sort of 'quick fix' solution to the problem with the earlier model that behaved too conservatively. However, in real-life OR projects one often has to resort to such 'quick fixes' because of lack of 
data, time or other resources or simply because a more sophisticated approach would not be worthwhile the effort. It is definitely a subject for further investigation, whether it would be worthwhile to deal with these matters in a theoretically perhaps more satisfactory way. Finally, another issue that could be raised is whether the demand parameters should be estimated from exponentially weighted historical data rather than estimated through the moving average type procedure that is suggested here.

\section{Inventory control}

The inventory control policy used by the distribution centre at Danfoss' RA-division is a base-stock policy with parameter (order-up-to level) $S$. The aim is to select $S$ so that a satisfactory order fill rate $(O F R)$ is achieved. The order fill rate is defined as the fraction of (the unknown) orders for which the whole order is available on time at the distribution centre. Before our common project, Danfoss used a standard heuristic method, essentially based on a cycle service level requirement, and the assumption that the lead-time demand distribution could be described by a normal distribution. However, this assumption was not fully satisfactory, because demands are often low frequent and individual customer orders differ in size. Furthermore, even if the normal distribution approximation did hold, as in the case of some high-volume SKUs, it was also clear that the service level requirement was not consistent with the preferred $O F R$ service measure. An OFR-type measure is also what is used for ex post performance measurement of the distribution centre operation.

Our mathematical model is a base-stock model assuming that all unknown orders have agreed delivery lead time $D T_{i}=0$ (as noted above, after the filtering procedure all advance-order information is suppressed), and all replenishment orders have a constant lead time $L$. Unfilled demand is backlogged, and furthermore any order that can not be filled immediately is assumed to be partially filled. The demand process is modelled as a compound renewal (Erlang) process with order size distribution $X$ and time between order request distribution $T$, where the distributions of $X$ and $T$ have been specified and estimated as described in Section 3. Let $D_{L}$ denote the aggregate demand during the replenishment lead time. Then $D_{L}$ has the probability distribution (Cox, 1962) 


$$
P\left(D_{L}=x\right)=\left\{\begin{array}{cc}
e^{k \lambda L} \sum_{j=0}^{k-1} \frac{(k \lambda L)^{j}}{j !} & x=0 \\
e^{k \lambda L} \sum_{m=1}^{x} P(X(m)=x) \sum_{j=0}^{k-1} \frac{(k \lambda L)^{j+m k}}{(j+m k) !} & x=1,2, \ldots
\end{array}\right.
$$

Note that in the case where $k=1$, this expression can be rewritten recursively, see Adelson (1966). The OFR service measure can now be determined in the following way. With probability $P\left(D_{L}=x\right)$ there will at the time of an order request be a net inventory of $S$ - $x$ units. Therefore, the whole order will be filled with probability $P(X \leq S-x)$. These two probabilities are independent. Hence, the $O F R$, as a function of $S$, is given by

$$
\operatorname{OFR}(S)=\sum_{x=0}^{S-1} P\left(D_{L}=x\right) P(X \leq S-x)
$$

For a given required level $\beta$ of the service measure $O F R$, we then find the smallest value of $S$ that satisfies $\operatorname{OFR}(S) \geq \beta$. Larsen and Thorstenson (2006) elaborate further on the OFR measure and specify in some detail how it is related to the standard (volume) fill rate and to some other service level concepts.

\section{Implementation and application}

We can now summarise the computational procedure for an update of the inventory control system parameters. Data are first extracted from the Business Warehouse system at Danfoss' RA-division. Next, the filtering procedure is conducted, and thereafter the parameters L, EmpM, EmpVar, $\lambda$, TBOmin, $\beta$, CosMax are estimated and inserted as inputs in a spreadsheet. Data for each SKU are in separate rows. The computational procedure to find order-up-to levels $S$ is programmed as a macro in Visual basic in Excel. The macro reads input data for one SKU at a time. Hereafter it estimates (or forecasts) the demand process, i.e. it specifies the distributions of $X$ and $T$, respectively. Finally, it carries out repeated computations of $\operatorname{OFR}(S)$ for incremental values of $S$ until $\operatorname{OFR}(S) \geq$ $S$. For an illustration of the computational procedure see Tables 1 and 2, where Table 1 contains the inputs, while the outputs are shown in Table 2.

$<$ Tables 1 and 2 about here $>$ 
The macro can compute base-stock levels for about 4000 SKUs in about one hour. Because the computational procedure for parameter updating is only intended to be used, say, once a month, this computational time does not appear to be an issue of major concern.

Regarding interpretation of the output from the computation, it is important to make users of the control tool aware of the fact that the computed service levels are expected values, corresponding to what would be observed in the long run. When performance is measured periodically on a short-term basis, the actual service levels obtained may of course differ considerably from the computed expectations for a specific SKU, especially for low frequent SKUs. The effect may be diminished, if the service levels are measured across groups of SKUs.

We have made comparisons between the results of our analytical inventory control model and the previous heuristic method used by the RA-division at Danfoss. In general, our analytical model does not produce overall lower base-stock values than those generated by the heuristic method. However, the primary objective of this project was not to achieve stock reductions per se, but to develop a consistent methodology for inventory control. The analytical model is obviously intended as an operational tool, which can provide management with guidelines for setting base-stock levels appropriately.

The model can also be applied as a tactical tool, conducting various sensitivity analyses, e.g. exploring the effects of reducing the variability of the unknown customer orders, increasing the fraction of known orders, reducing lead times, etc. It may also be helpful in the reverse process of identifying required parameters to reach certain inventory-related performance goals. The results of such analyses might be useful as a guide for ranking various demand planning activities that Danfoss' RA-division is considering. This distinction between operational and tactical purposes underlines the phrase it is better to prevent than to cure. Solely focusing on operational issues represents the cure. However, having a tool which can also quantify future possible scenarios also qualifies it to be a vital part of the preventive work. 


\section{Conclusions and discussion}

We have presented the development and design of an analytical model for inventory control as well as indicated issues related to its implementation and usage. As in any ORrelated project, the system dealt with has to be delineated, modelling assumptions have to be imposed and compromises have to be struck between what is desirable from a theoretical point of view and what is possible when considering the practical realities. The acid test for any practicable decision support tool for planning and control is whether it can contribute to improved business performance.

Obviously, there is room for further improvements of the model. For example, at the present stage of development, there are no elements of coordination included either between the various SKUs or between the different tiers of the supply chain. Here, we will comment on two other relevant issues, namely the possible incorporation of advance order information and the ability to cope with seasonality and trends.

As commented previously, after completing the filtering process all advanced-order information is suppressed. It is, however, possible to deduce mathematically an expression for $O F R(S)$, under the assumption that orders are served according to the date of their request (so that customers who offer a long agreed delivery lead time are rewarded). However, this requires information about the distribution of advance orders. Related to this issue is also the structure of the optimal inventory control policy. In combination with the renewal demand process it might be of particular interest to consider some kind of delayed ordering policy rather than the pure base-stock policy, which is not the optimal policy in general for this case, as noted in Section 2.

Furthermore, for some SKUs there is a substantial element of seasonality or trend in the demands. For those SKUs the recommendations of our model will lag behind, as there is no provision for such elements in the present version of the model other than frequent parameter updates. Most likely, it is possible to construct an improved version of our model to, at least partially, incorporate these elements in a more sophisticated way. Obviously, such developments will come at the expense of requirements for further inputs and computations. We are currently in the process of exploring the possibilities for making (simple) modifications of the model to deal with these issues. 


\section{References}

Adelson, R M (1966). Compound Poisson distributions. Opl Res $Q$ 17: 73-75

Axsäter, S (2006). Inventory Control. $2^{\text {nd }}$ edition, Springer's International Series

Bradley, J R and Robinson, L W (2005). Improved base-stock approximations for independent stochastic lead times with order crossover.

Manuf Service Opns Mngt 7: 319-329

Burgin, T A (1975). The gamma distribution and inventory control.

Opl Res $Q$ 26: 507-524.

Chen, F Y and Krass, D (2001). Inventory models with minimal service level constraints. Eur J Opl Res 134: 120-140

Croston J D (1972). Forecasting and stock control for intermittent demands. Opl ResQ 23: 289-303

Cox, D R (1962). Renewal Theory. Methuen: London

Feeney, G J and Sherbrooke, C C (1966). The $\left(s^{-1}, s\right)$ inventory policy under compound Poisson demand, Mngt Sci 12:391-41

Feller, W (1966). An Introduction to Probability Theory and Its Applications, Vol. II, J. Wiley \& Sons, New York

Hadley, G and Whitin, T M (1963). Analysis of Inventory Systems. Prentice-Hall, Englewood Cliffs.

Hariharan R and Zipkin, P H (1995). Customer-order information, lead times, and inventories. Mngt Sci 41: 1599-1607

Hua, Z S, Zhang, B, Yang, J, and Tan D S (2007). A new approach of forecasting intermittent demand for spare parts inventories in the process industries.

J Opl Res Soc, 58: 52-61

Johnston, F R and Boylan, J E (1996). Forecasting for items with intermittent demand. J Opl Res Soc 47: 113-121 
Johnston, F R, Boylan, J E, and Shale, E A (2003). An examination of the size of orders from customers, their characterization and the implications for inventory control of slow moving items. J Opl Res Soc 54: 833-837

Larsen, C and Thorstenson, A (2006). A comparison between the order and the volume fill rate for a base-stock inventory control system under a compound renewal demand process. Working paper, Logistics/SCM Research Group, Department of Business Studies, Aarhus School of Business

Law, A M and Kelton, W D (1991). Simulation Modeling $\&$ Analysis, $2^{\text {nd }}$ ed. McGrawHill, Inc.: New York, NY

Marklund, J (2006). Controlling inventories in divergent supply chains with advanceorder information. Opns Res 54: 988-1011

Matheus, P and Gelders, L (2000). The $(R, Q)$ policy subject to a compound Poisson demand pattern. Int J Prod Econ 68: 307-317

Park, C (2007). An analysis of the lead time demand distribution derivation in stochastic inventory systems. Int J Prod Econ 105: 263-272

Schneider, H (1981). Effect of service-levels on order points or order-levels in inventory models. Int J Prod Res 19: 615-631

Silver, E A, Pyke, D F, and Peterson, R (1998). Inventory Management and Production Planning and Scheduling, $3^{\text {rd }}$ edition. John Wiley \& Sons: New York, NY

Smith, M A J and Dekker, R (1997). On the $(S-1, S)$ stock model for renewal demand processes. Prob Engng Inf Sci 11: 375-386

Song, J-S (1998). On the order fill rate in a multi-item base-stock inventory system. Opns Res 46: 831-845

Tempelmeier, H (2000). Inventory service levels in the customer supply chain. OR Spektrum 22: 361-380

Tyworth, J E, Guo, Y, and Ganeshan, R (1996). Inventory control under gamma demand and random lead time. J Bus Log 17: 291-304 
Ward, J B (1978). Determining reorder points when demand is lumpy.

Mngt Sci 24: 623-632

Willemain, T R, Smart, C N, and Schwarz, H F (2004). A new approach to forecasting intermittent demand for service parts inventories. Int J Forecast 20: 375-387

Zipkin, P H (2000). Foundations of Inventory Management. McGraw-Hill, Inc.: Boston, MA 


\section{Tables}

\begin{tabular}{|c|c|c|c|c|c|c|c|}
\hline Material & $\begin{array}{c}\text { Lead } \\
\text { time }\end{array}$ & $\begin{array}{r}\text { Average } \\
\text { order size } \\
\end{array}$ & $\begin{array}{r}\text { Variance of } \\
\text { order size } \\
\end{array}$ & $\begin{array}{l}\text { Average number } \\
\text { of orders per day }\end{array}$ & TBOmin & $\begin{array}{r}\text { Required } \\
\text { OFR } \\
\end{array}$ & CosMax \\
\hline $003 N 2107$ & 13 & 15.500 & 0.500 & 0.008 & 79 & 0.98 & 16 \\
\hline $003 N 2113$ & 6 & 24.000 & 375.000 & 0.042 & 0 & 0.90 & 60 \\
\hline $003 N 2114$ & 13 & 11.444 & 280.540 & 0.138 & 0 & 0.98 & 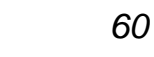 \\
\hline $003 N 2119$ & 13 & 38.429 & 133.187 & 0.054 & 2 & 0.98 & 57 \\
\hline $003 N 2125$ & 13 & 3.067 & 10.781 & 0.058 & 2 & 0.98 & 1 \\
\hline $003 N 2128$ & 6 & 25.000 & 0.000 & 0.004 & 50 & 0.90 & 2 \\
\hline $003 N 2132$ & 6 & 2.670 & 11.611 & 1.338 & 0 & 0.98 & 3 \\
\hline $003 N 2162$ & 6 & 3.816 & 35.538 & 4.442 & 0 & 0.98 & 5 \\
\hline $003 N 2164$ & 6 & 16.941 & 163.059 & 0.065 & 1 & 0.90 & \\
\hline
\end{tabular}

Table 1: Input data for the macro to compute inventory control parameters.

\section{OUTPUT:}

\begin{tabular}{|lrr|rrr|}
\multicolumn{2}{c|}{ Demand distribution characteristics } & \multicolumn{3}{c}{ Output for order fulfilment } \\
Type & Form parameter & Prob. parameter & Order-up-to level & Actual OFR & $k$ \\
\hline Binomial & 17.000 & 0.9655 & 17 & 1.000 & 9 \\
\hline NegBin & 4.424 & 0.8387 & 54 & 0.901 & 1 \\
NegBin & 1.661 & 0.8628 & 87 & 0.981 & 1 \\
NegBin & 79.911 & 0.3190 & 103 & 0.980 & 3 \\
\hline NegBin & 0.851 & 0.7083 & 15 & 0.985 & 3 \\
Binomial & 25.000 & 1.0000 & 25 & 1.000 & 4 \\
NegBin & 0.280 & 0.8562 & 57 & 0.981 & 1 \\
NegBin & 0.242 & 0.9207 & 195 & 0.981 & 1 \\
NegBin & 1.727 & 0.9022 & 41 & 0.902 & 2
\end{tabular}

Table 2: Output from the macro given the given input in Table 1. The first three columns contain information about the chosen order-size distribution In the next two columns, the optimal order-up-to level $(S)$ is computed as well as the $O F R(S)$. Finally, the $k$ parameter of the Erlang inter-arrival distribution is shown. 


\section{Working Papers from Logistics/SCM Research Group}

L-2007-02 Christian Larsen, Claus Hoe Seiding, Christian Teller \& Anders Thorstenson: An inventory control project in a major Danish company using compound renewal demand models.

L-2007-01 Christian Larsen: The $Q(s, S)$ control policy for the joint replenishment problem extended to the case of correlation among item-demands.

L-2006-11 Daniele Pretolani, Lars Relund Nielsen \& Kim Allan Andersen: A note on "Multicriteria adaptive paths in stochastic, time-varying networks".

L-2006-10 Lars Relund Nielsen, Kim Allan Andersen \& Daniele Pretolani: Bicriterion a priori route choice in stochastic time-dependent networks.

L-2006-09 Christian Larsen \& Gudrun P. Kiesmüller: Developing a closed-form cost expression for an $(R, s, n Q)$ policy where the demand process is compound generalized Erlang.

L-2006-08 Eduardo Uchoa, Ricardo Fukasawa, Jens Lysgaard, Artur Pessoa, Marcus Poggi de Aragão, Diogo Andrade: Robust Branch-Cut-and-Price for the Capacitated Minimum Spanning Tree Problem over a Large Extended Formulation.

L-2006-07 Geir Brønmo, Bjørn Nygreen \& Jens Lysgaard: Column generation approaches to ship scheduling with flexible cargo sizes.

L-2006-06 Adam N. Letchford, Jens Lysgaard \& Richard W. Eglese: A Branch-andCut Algorithm for the Capacitated Open Vehicle Routing Problem.

L-2006-05 Ole Mortensen \& Olga W. Lemoine: Business integration between manufacturing and transport-logistics firms.

L-2006-04 Christian H. Christiansen \& Jens Lysgaard: A column generation approach to the capacitated vehicle routing problem with stochastic demands.

L-2006-03 Christian Larsen: Computation of order and volume fill rates for a base stock inventory control system with heterogeneous demand to investigate which customer class gets the best service.

L-2006-02 Søren Glud Johansen \& Anders Thorstenson: Note: Optimal base-stock policy for the inventory system with periodic review, backorders and sequential lead times.

L-2006-01 Christian Larsen \& Anders Thorstenson: A comparison between the order and the volume fill rates for a base-stock inventory control system under a compound renewal demand process. 
L-2005-02 Michael M. Sørensen: Polyhedral computations for the simple graph partitioning problem.

L-2005-01 Ole Mortensen: Transportkoncepter og IT-støtte: et undersøgelsesoplæg og nogle foreløbige resultater.

L-2004-05 Lars Relund Nielsen, Daniele Pretolani \& Kim Allan Andersen: K shortest paths in stochastic time-dependent networks.

L-2004-04 Lars Relund Nielsen, Daniele Pretolani \& Kim Allan Andersen: Finding the $K$ shortest hyperpaths using reoptimization.

L-2004-03 Søren Glud Johansen \& Anders Thorstenson: The $(r, q)$ policy for the lostsales inventory system when more than one order may be outstanding.

L-2004-02 Erland Hejn Nielsen: Streams of events and performance of queuing systems: The basic anatomy of arrival/departure processes, when focus is set on autocorrelation.

L-2004-01 Jens Lysgaard: Reachability cuts for the vehicle routing problem with time windows. 
ISBN 87-7882-182-7

Department of Business Studies

Aarhus School of Business

University of Aarhus

Fuglesangs Allé 4

DK-8210 Aarhus V - Denmark

Tel. +4589486688

Fax +4586150188

www.asb.dk 